\title{
Recommendations on the Internet of Things: Requirements, Challenges, and Directions
}

\author{
Lina Yao, UNSW Australia \\ Xianzhi Wang, University of Technology Sydney \\ Quan Z. Sheng, Macquarie University \\ Schahram Dustdar, TU Wien \\ Shuai Zhang, UNSW Australia
}

\begin{abstract}
The Internet of Things (IoT) is accelerating the growth of data available on the Internet, which makes the traditional search paradigms incapable of digging the information that people need from massive and deep resources. Furthermore, given the dynamic nature of organizations, social structures, and devices involved in IoT environments, intelligent and automated approaches become critical to support decision makers with the knowledge derived from the vast amount of information available through IoT networks. Indeed, IoT is more desirable of an effective and efficient paradigm of "proactive discovering" rather than postactive searching. This article discusses some of the important requirements and key challenges to enable effective and efficient Thing-of-Interest recommendation, and provides an array of new perspectives on IoT recommendation.
\end{abstract}

\section{Background and Motivation}

The Internet of Things (IoT) is increasingly contributing to the growth of data on the Internet with advanced capabilities of accessing, connecting, and managing physical devices $[6,8,14,19]$. The traditional search paradigms have found it more difficult than ever to obtain such information as to cater a specific request from a massive and deep resource base including the IoT [16]. Moreover, the dynamic nature of devices, organizations, and social structures involved in the IoT calls for intelligent and automated approaches to knowledge mining and decision-making based on the vast amount of information available through IoT networks. It is, therefore, more desirable to provide an effective and efficient paradigm for proactive discovering rather than postactive searching for IoT resources and information. This way, instead of a user with a certain information need searching for, and retrieving from, the relevant resources, a system built on the proactive discovery paradigm will push the most relevant resources to the user, based on the user's history of interactions with both resources and other users.

To realize proactive discovery on the future Internet, this article explores the topic of thing-of-interest recommendation [17]. We stress that things recommendation is a crucial step towards promoting and taking full advantage of the capabilities of IoT. A recommendation approach for the IoT holds the potential to benefit individuals, businesses, and the society on a daily basis [13]. It cannot only reduce users' effort on discovering the relevant things by exploring their (hidden) preferences and interests but also assist users in making better choices on selecting things by pre-evaluating and ranking the recommendation results. However, despite the significant advances, things recommendation is much more complex than traditional recommender systems like recommending books or movies, and the current generation of recommender systems still 
requires further improvements to make recommendation methods more effective and applicable to an even broader range of IoT applications. Physical things in reality have multiple unique attributes (e.g., physical things have states such as in use or not in use; expired or not expired), and recommendations in IoT is usually more spontaneous, context dependence and monopolistic under certain circumstances when compared with traditional recommendations. When a certain thing is in use, it generally cannot be used simultaneously by another user. Under this circumstance, a recommender system can refer the user to a list of similar things that are available [18].

Consider Thomas, a 77 year-old frail man who lives alone in a small apartment with a network of deployed sensors. The system built upon these connected sensors automatically monitors and analyzes Thomas' activity patterns, and identifies any incidents that might indicate an emergent or deteriorating health/mental condition before it becomes critical. Then, the appropriate package of medical intervention and wellness management are provided proactively.

IoT recommendation involves observations, perceptions, knowledge discovery, and intelligent reasoning. A person's daily activities may include various actions such as taking pills regularly for his hypertension, video chatting with his children and friends, and taking a walk in the morning. His physical index such as sugar level, blood pressure, food intake, exercise, and sleep, could be continually monitored with sensors (e.g., Apple watch or medical wrist). An important related question centers on how to automatically recommend the thing-of-interest (TOI) that the person might need (e.g., taking pills for controlling his blood pressure, having a walk for keeping him fit, and "face-timing" with his daughter) timely and precisely. The recommendation is especially important in the case that we can automatically identify any changes from the daily data that might indicate an emerging or deteriorating health or mental condition (e.g., Alzheimer's disease) before it becomes critical. The observations and perceptions from the physical world, social world and digital world are strongly influenced by our cognition, behaviors, and background knowledge, but can still help us derive and identify the patterns and perceptions.

By analyzing these observations and interactions in the context of fully connected physical-social-digital Internet of Things and building up the relationships between them, we can form a knowledge graph of things and orient ourselves towards the subsequent actions. Then, decisions regarding which action to be taken can be recommended based on the evidence and knowledge derived.

The distribution, scale, heterogeneity, multimodality, streaming data, and dynamicity of IoT environments imply that existing solutions for discovering, accessing, and using the information on the Internet is calling for more effective and efficient paradigm of proactive discovering, rather than post-active searching, in which a user who has certain information needs will search on the Internet for related resources, while the concept of "discovery" refers to the ability to push relevant and related resources to the user, especially according to the metadata collected from the user's historical activities in terms of activities on resources or with other people. Effective recommendation is a crucial step for promoting and taking full advantage of the IoT to foster the development of practical and large-scale dynamic IoT applications, where it benefits the individuals, businesses, and society on two main aspects. On the one hand, it can deliver relevant things and related services provided by thing-of-interest (i.e., things that users might need or be interested in) to users based on their preferences and interests. On the other hand, it can also serve to optimize the time and cost of using IoT in a specific domain.

The following are some of the reasons that cause the additional complexity in things recommendation. First, physical things have multiple unique attributes. When a certain 
thing is in use, it cannot be used simultaneously by another user. Under this circumstance, a recommender system can refer the user to a list of similar things that are available. Second, recommendation in IoT is more spontaneous, context dependence and monopolistic under certain circumstances compared with traditional recommendations. Consequently, IoT recommendation is much more complex than traditional recommender systems like recommending books or movies. This article discusses the requirements and challenges, and identifies the directions for developing a robust and comprehensive recommendation solution for the IoT.

\section{Related Work}

Proposals vary from an enrichment of traditional recommender systems to a radical change in paradigm. Several recent works provide methods and techniques for recommending Thing-of- Interest in the IoT scenario. Generally, recommender systems can be used to assist groups of users to select services in IoT-enriched environments. Most existing research falls into three categories: content-based filtering, collaborative filtering, and link-based systems [11]. Chirila et al. [3] present a broker-based architecture for service selection which facilitates devices to specify both functional and nonfunctional requirements in the IoT context. Mashal et al. [10] introduce the concept of service recommender systems in IoT by a formal model and then propose a graph-based recommender system that uses hyper-edges to connect users, objects, and services. They further study the usefulness of traditional recommendation schemes and the hybrid approaches based on these schemes on IoT service recommendation using existing wellknown metrics.

Considering inter-things relations, Forestiero et al. [4] build a distributed recommendation system in an IoT environment where things are represented through bit vectors that encode similar things into similar bit vectors. They use cyber agents to manage and exchange thing descriptors to support an efficient things recommender system. Inter-person relations are another important aspect of IoT recommendation. Chen et al. [2] propose a scheme of access service recommendation based not only on the past performance (inherent constraints and factors that influence the security and stability of IoT networks) but also on the social relationship among things and the energy status of nodes. Given the multi-aspect relations, Yao et al. [17] propose a unified probabilistic factor-based framework by fusing user-thing relations, user-user relations, and thingthing relations across heterogeneous entities of IoT and develop a hypergraph to model things' spatiotemporal correlations to make things recommendations.

Further development includes a user-based collaborative filtering approach [7], which considers member organization for a new user group. This approach selects neighboring user groups that are similar to the new group based on combinations of various grouplevel similarity metrics such as the group size-based, common member-based, and member preference-based metrics. Another direction of research is IoT-enabled contextaware recommendation [1], which accommodates user's needs with location-dependent multimedia information available in a mobile environment.

\section{Challenges from Recent IoT Progress}

We identify several challenges for an IoT recommender system, arising from the recent progress in the IoT-related technologies and systems. We envision a recommender system that can deal with the challenges by undergoing the following transformations.

From static, contextless to dynamic, context-aware recommendation. IoT resources are inherently unreliable and have underlying relations [18]. The IoT resources are more 
ad-hoc compared with traditional resources like documents and images. Due to varying locations, device limitations, and transmission constraints of IoT resources, there might be noises, missing values, discrepancies, or even conflicts in their data. For trustworthy applications upon these data, there should be some means of evaluating and predicting the performance and data quality of IoT resources. Also, it is important to investigate their underlying relations, e.g., whether they provide similar functionalities, whether they are deployed at adjacent locations, or whether they are frequently used together, in order to be able to use these resources effectively in future applications. Thing-of-interest are usually associated with descriptions, categories, or social tags to describe their attributes of functionality or non-functionality. However, unlike traditional recommendation (i.e., recommending a movie), the textual information associated with things is usually incomplete and ambiguous. In addition, this incompleteness is also caused when sensors malfunction or are disconnected, or there is partial coverage due to factors such as communication or network limitations. The services provided by IoT devices are contextaware, e.g., they should be appropriate to be recommended and be available (e.g., not expired, not disconnected). In addition, IoT data are inherently multi-sourced, multifaceted, heterogeneous, as well as of varying quality [1]. This makes it more complicated to describe IoT resources than traditional Web resources such as documents, images or video clips. Given various devices deployed at different locations and capturing different types of information about the same or different environmental objects, they may use different communication protocols and vendor-specific propriety network solutions. The captured data may also have different formats and of varying accuracy. To enable effective exploitation to these data, the data need to be well-organized and described using unified representations, with their quality information attached.

From homogeneous recommendation to heterogeneous recommendation. IoT resources are typically used collectively to produce large data in dynamic environments $[17,19]$. Differing from traditional recommendation problems, typical IoT applications require using multiple types of IoT resources, many of which providing the same types of data to make trustworthy decisions. This calls for novel recommendation methods to recommend heterogeneous IoT resources (rather than a set of homogeneous resources such as a set of books or movies) as a group, and adapt to the constantly changing status of things and always present the most up-to-date recommendation results. Also, given accidental, periodical or continuous data generated by IoT resources, efficient online recommendation and optimization techniques are required. A challenging point related to the heterogeneity is that humans inevitably develop a sense of relationships between objects, some of which are based on their appearance. Some objects might be alternatives to each other (such as two pairs of jeans) while others may be complementary (such as a pair of jeans and a matching shirt). This requires a hybrid recommendation approach that can effectively leverage the heterogeneous relationships among things, as opposed to the traditional multi- and cross-domain recommendation.

From accuracy-focused recommendation to security- and privacy-aware recommendation. As sensors from the Internet of Things start to infuse people's everyday life, companies are highly interested to receive such data from their users for more effective recommendation of their products and services, and sharing private data with a company might be beneficial for both the company and customers. When more and more personal information is collected for more accurate recommendation, it brings great privacy concerns to the public. Several factors have an influence on consumers' privacy concerns, such as perception of risk, desire for control, lack of trust, anxiety, and loss of comfort. Besides, the service providers (e.g., IoT devices) could suffer from attacks launched by malicious users who want to bias the recommendations. Generally, 
the trust relationships in IoT recommendation are multi-faceted and heterogeneous, and the privacy of the users of IoT applications should be guaranteed. Therefore, a practically effective and efficient privacy-preserving mechanism to guarantee the anonymity of a customer and the protection of personal data is desirable. However, none of the current security measures and privacy preserving techniques can guarantee the anonymity of a customer, the safety, or controllability of users' data profile in large-scale distributed heterogeneous environments like an IoT environment. Take the dominant privacypreserving techniques as an example. Cryptography often incurs significant computational overhead even for simple computations on a small dataset; Perturbation lacks the theoretical guarantee; and differential privacy is usually designed for a specific scenario and cannot defend against various types of possible attacks and data leakage risks when complex computations are performed on large data sets as conducted by a recommender system in an IoT environment.

From a singleton interface to an interactive, multi-modal interface. A multimodal interface of a recommender system includes the presentations and functionalities to support the interactions facilitated by users' speech, gestures, eye movement, or a BrainComputer Interface [13]. An IoT-based system enables users to interact with physical things in a broad range of ways by collecting, analyzing the environmental data and helping people or hardware systems make automatic reactions or informed decisions. Therefore, the interfaces of traditional recommender systems such as Web browsers or mobile phone interfaces fall insufficient to support such enriched interactions through the use of audio, visual and physiological signals. Another requirement arise from the situations where the user does not agree with the recommendation results and may wish to update or alter the recommended item by herself. This way, the interfaces not only serve as a way of providing complex information but also a media for the user to gain an easy understanding of the recommendation process and results, as well as to accomplish quick manipulation or intervention of the recommendation procedure.

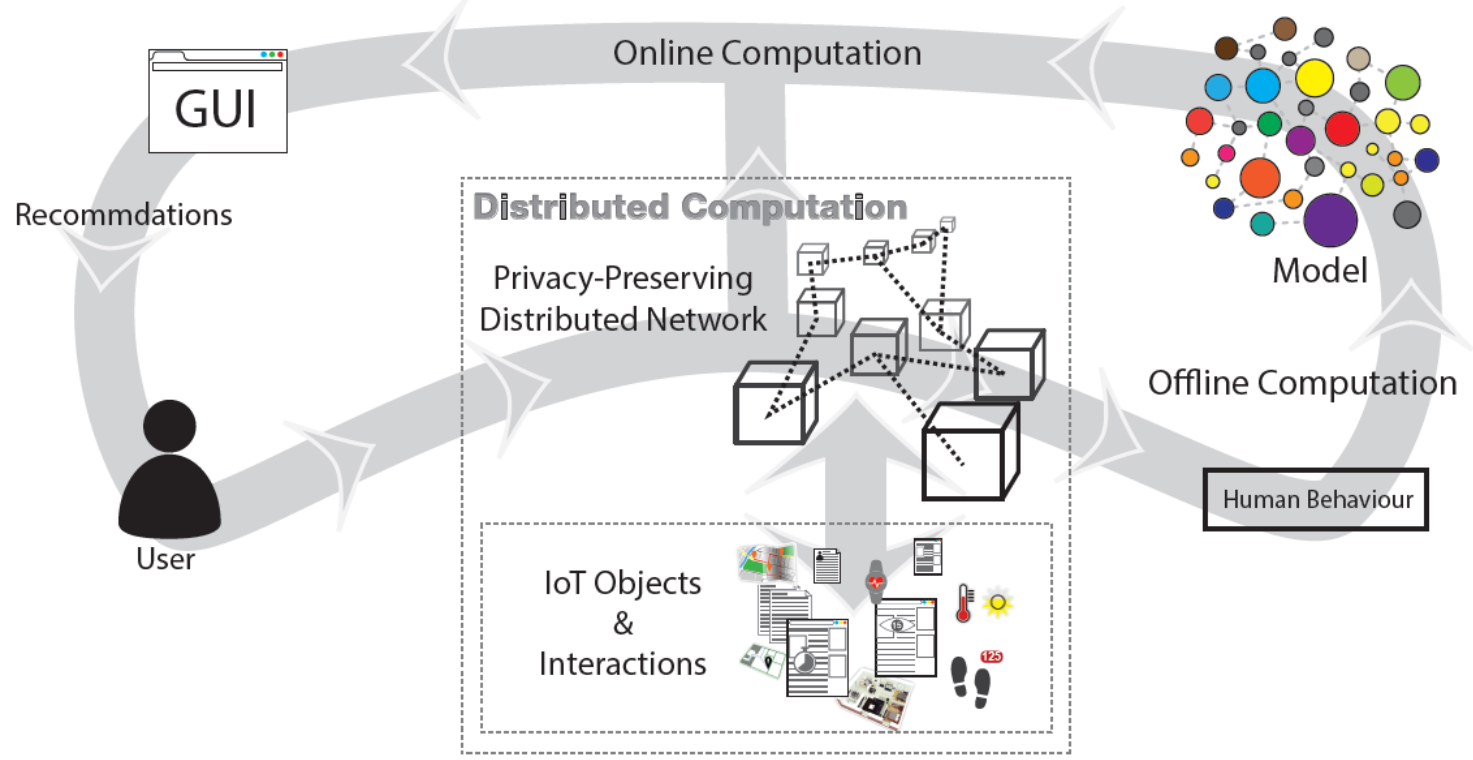

Figure 1: The conceptual framework for IoT recommendation

From centralized recommendation to distributed recommendation. The IoT data are highly likely to be located on distributed devices and require considerable efforts to be aggregated for centralized analysis. The centralization not only demands high network 
traffic but also cause the trained models (esp., those complex models that require a large amount of time for re-training) based on the aggregated data too general to cater to each end-user's individual demands. Under this situation, a recent trend in the recommendation research is to deploy recommendation algorithms run on users' end-devices like mobile phones or portable IoT devices to provide context-aware services other than a centralized server. It is, therefore, necessary to develop the software and hardware to build modular gateway solutions for managing devices and data in IoT. The whole system should support the local management of devices and data, app development, and include security features that allow users to share data in a trusted way. The recommendation technology should progressively attract the interest of new application domains as a valuable solution to increase system autonomy and efficiency. Modern embedded systems involved in IoT scenarios can exploit the advantages offered by context-aware and profile-driven recommendations. The objective is to recommend things of interest to the end-user based on not only preferences of local users but also the commonsense inferred from the history of the mass users. The emerging distributed computing paradigms such as fog/edge computing [6] can also be leveraged to support the local and global knowledge discovery for the effective and efficient distributed recommendation.

\section{A Conceptual Framework for IoT Recommendation}

In view of the above challenges posed by the IoT environment, we propose a conceptual framework as a preliminary, yet holistic solution to the IoT recommender systems (Figure 1). The aim is to bring together different computational resources and analytical capabilities according to the data life-cycle of an IoT application. The framework includes four layers (or components) as the following:

- Distributed computing layer. This layer provides the storage, communication, as well as security and privacy support for high-level applications such as recommender systems on top of the IoT infrastructure. Besides the recommendation accuracy, IoT environments raise more concerns than traditional recommender systems, such as (distributed) cyberattacks, information leakage, or source tracking during both data sharing, transmission, and mining. For example, depending on the different tasks and IoT networks, we may want to protect the location of data sources, the identification of data sources, the information content, or the performance profiles of sources. The dominant encryption techniques such as homomorphic encryption [5] and multiparty computation [9] can help prevent data leakage, while blockchain-based solutions can be developed to provide undeniability and accountability to all transactions occurring in the whole system.

- Offline computation layer. This layer trains machine learning or deep learning models to learn the patterns in human activities or human preferences on high performance computing facilities such as cloud data centers. The learning follows either centralized or distributed learning paradigms such as federated learning, depending on the scale of data and capabilities of computing resources. The trained model should comprehensively consider the heterogeneous relations among things, users, as well as between things and users, depending on the specific scenarios. The offline model can serve as a basis for customization by the online computation model or as a knowledge base that provides the overall guidance to the recommendations to different individual tasks.

- Online computation layer. This layer differs from the offline computation layer in conducting real-time computation to provide prompt feedbacks to dynamic user context or upcoming events. This requires using not only highly efficient models to deliver recommendation results within an acceptable time frame but also highly 
customizable models which make personalized recommendation of a single item, a set of items, or a list of items to either an individual user or a group of users. The emerging edge/fog computing paradigms and the advances in embedded system technologies offer opportunities for achieving high performance learning on individual devices.

- User interface layer. This layer is the application interface where users can input their requirement specifications, view the search or recommendation results, manipulate the exploratory process of gaining the satisfactory results, and provide feedbacks to the recommendation mechanism. Besides the interactive capability, the user interface can serve as a place to show visual interpretations [20] or evidencebased explanations of the recommendation results, helping users better understand the results.

Back to the smart home scenario we mentioned earlier, under the new recommendation frame- work for the IoT, the sensor signals of numerous elderly people like Thomas are sent to remote cloud servers, where the data are integrated and used to train various modeling concerns various activities that occur in each home environment. The edge servers or embedded devices store the people's real-time context information and make real-time judgment on the event happening in each home as well as the necessary actions to take from the system's side. Thomas will be notified immediately as the back-end system makes new arrangement for him, and he can intervene as necessary whenever he has the ability and the will to do so.

\section{Future Directions}

In retrospect of the challenges against the existing and new trends in the recommendation techniques for the IoT, we envision several promising directions for the future research.

Deep learning in IoT recommendation. Machine learning has been extensively used for various tasks including thing-of-interest recommendations in the IoT environments. Deep learning emerges and draws increasing attentions in various occasions for two main reasons. First, as the data become more complex, there is a pressing need to analyze data that cannot be easily understood by humans. It could also be impossible for human to manually extract and define the features to train a model. Another aspect that contributes to the complexity is the diverse relations among different objects in the data [18]. All the above challenges demand more comprehensive methods such as a deep learning model for the learning task. Second, while machine learning techniques suffice in many cases that involve structured or semi-structured data, they often fall reluctant to help understand or process unstructured multimedia data, such as audio signals and visual images. More sophisticated deep-learning technologies, instead, can use neural networks to capture and understand complex environments from which humans cannot easily find clues based on mere intuition. Deep learning can be deployed to an IoT environment either on a central server as a cloud-centric learning unit [12] or on edge computing facilities or IoT devices (i.e., end-user devices) to provide customized, efficient recommendation [8]. A main challenge regarding deploying deep learning on IoT devices is the demanding real-time computation requirements. The possible solutions include offloading deep learning to the cloud or migrating deep learning platforms to the edge (e.g., IoT devices) [15].

Explanation for IoT recommendation. Interpretability has drawn increasing attentions in the recommender systems research as more complicated models (e.g., deep learning models) are employed in recommendation tasks [20]. The aim of achieving explainable or interpretable recommendation is to provide an intuitive and proof-supported explanation of the recommendation results in order to better convince users the rationale of the results or help them gain more insights towards the factors behind the 
recommendation. Until now, we have not seen any research, to the best of our knowledge, on promoting the interpretability of recommender systems for an IoT environment. An important observation from the related previous research is that a model's interpretability strongly relies on the application domain and usage of content information. For example, different mediums such as user reviews, images, or abstractive tips can be linked to prediction results to interpret how they contribute to different possible prediction results through the use of attention mechanisms in neural networks.

Combining searching for more powerful IoT recommendation. Traditionally, search engines are mainly used by human users entering keywords or questions [16], and a recommendation approach surpasses the search-based approach in actively discovering and leveraging both user's hidden preferences (some of which may not be explicitly expressed by humans) and implicit relations among different entities (e.g., things to things correlation) involved in the recommendation. We believe combining searching with recommendation could deliver better user experience and recommendation performance. It is also a good way to mitigate the cold-start problem faced by most recommender systems, as more and more devices are becoming available on the Internet. We envision recommendation as an enhancement to the traditional search by providing additional guidance to users' exploration to their things of interest. This way, the search engine serves not only as a things locator but also a route showing only the most promising paths to the satisfactory results. Conversely, searching can serve as a semantically sound clue to assist more accurate recommendations. Enabling semantic analysis to the search inputs is a promising direction for research. The related techniques to improve the search and recommendation efficiency include efficient indexing (to quickly locate data without having to scan through all the records), attribute ranking rules (query-dependent and query-independent ranking of search results), and fuzzy query matching.

Blockchain-supported distributed recommendation. Internet of Things recommendation imposes harsher constraints on the data/device security and privacy as IoT data are comparatively more sensitive to both users and devices. Current solutions are unable to guarantee cryptographic secureness. Recently, blockchain-based approaches have been proved the effective solutions to cryptographically guarantee the proper usage of personal data. The core component is a decentralized peer-to-peer network that allows storing encrypted data in a tamper-proof way and runs secure computations while only the data owner has access to the raw data. A potential costumer can allow a company to apply a recommendation algorithm without disclosing her pro- file. The users never lose control of their private data and can terminate the business relationship at any time. Fraud and misuse are no longer possible, because the involved companies or data consumers would never get the raw data.

\section{Conclusion}

We have reviewed the research on Thing-of-Interest (TOI) recommendation and the related techniques in the Internet of Things (IoT) environments. We highlight the unique challenges brought by IoT and envision a conceptual framework for enabling the effective TOI recommendation for the IoT, followed by discussing several future directions that need more research efforts from the community. Through this work, we hope to provide a reference solution to the next-generation smart IoT-based systems and give researchers a quick grasp of the state-of-the-art and promising research challenges in this important area.

\section{Acknowledgments}

Lina Yao's work is partially supported by Australian Research Council DECRA grant 
DE160100509. Xianzhi Wang's work is partially supported by Australian Research Council DECRA grant DE180100251. Quan Z. Sheng's work is partially supported by Australian Research Council Future Fellowship grant FT140101247.

\section{References}

1. Flora Amato, Antonino Mazzeo, Vincenzo Moscato, and Antonio Picariello. A Recommendation System for Browsing of Multimedia Collections in the Internet of Things. In Internet of Things and Inter-cooperative Computational Technologies for Collective Intelligence, pages 391-411. Springer, 2013.

2. Zhikui Chen, Ruochuan Ling, Chung-Ming Huang, and Xu Zhu. A Scheme of Access Service Recommendation for the Social Internet of Things. International Journal of Communication Systems, 29(4):694-706, 2016.

3. Stefana Chirila, Camelia Lemnaru, and Mihaela Dinsoreanu. Semantic-based IoT Device Discovery and Recommendation Mechanism. In 2016 IEEE 12th International Conference on Intelligent Computer Communication and Processing (ICCP), pages 111-116. IEEE, 2016.

4. Agostino Forestiero. Multi-agent Recommendation System in Internet of Things. In 17th IEEE/ACM International Symposium on Cluster, Cloud and Grid Computing (CCGRID), pages $772-775,2017$.

5. Craig Gentry. Fully Homomorphic Encryption Using Ideal Lattices. In $41^{\text {st }}$ Annual ACM Symposium on Theory of Computing (STOC), pages 169-178, 2009.

6. Marjan Gusev and Schahram Dustdar. Going Back to the Roots - The Evolution of Edge Computing, an IoT Perspective. IEEE Internet Computing, 22(2):5-15, 2018.

7. Jin-Seo Lee and In-Young Ko. Service Recommendation for User Groups in Internet of Things Environments Using Member Organization-based Group Similarity Measures. In 2016 IEEE International Conference on Web Services (ICWS), pages 276-283. IEEE, 2016.

8. He Li, Kaoru Ota, and Mianxiong Dong. Learning IoT in Edge: Deep Learning for the Internet of Things with Edge Computing. IEEE Network, 32(1):96-101, 2018.

9. Yehuda Lindell and Benny Pinkas. A Proof of Security of Yao's Protocol for Two-party Computation. Journal of cryptology, 22(2):161-188, 2009.

10. Ibrahim Mashal, Tein-Yaw Chung, and Osama Alsaryrah. Toward Service Recommendation in Internet of Things. In 2015 Seventh International Conference on Ubiquitous and Future Networks, pages 328-331. IEEE, 2015.

11. David W McDonald. Ubiquitous Recommendation Systems. Computer, 36(10):111-112, 2003.

12. Mehdi Mohammadi, Ala Al-Fuqaha, Sameh Sorour, and Mohsen Guizani. Deep Learning for IoT Big Data and Streaming Analytics: A Survey. IEEE Communications Surveys \& Tutorials, 20(4):2923-2960, 2018.

13. Gunar Schirner, Deniz Erdogmus, Kaushik Chowdhury, and Taskin Padir. The Future of Humanin-the-loop Cyber-physical Systems. Computer, (1):36-45, 2013.

14. Quan Z. Sheng, Yongrui Qin, Lina Yao, and Boualem Benatallah. Managing the Web of Things: Linking the Real World to the Web. Morgan Kaufmann, 2017.

15. Jie Tang, Dawei Sun, Shaoshan Liu, and Jean-Luc Gaudiot. Enabling Deep Learning on IoT Devices. Computer, 50(10):92-96, 2017.

16. Nguyen Khoi Tran, Quan Z Sheng, Muhammad Ali Babar, and Lina Yao. Searching the Web of Things: State of the Art, Challenges, and Solutions. ACM Computing Surveys (CSUR), 50(4):55, 2017.

17. Lina Yao, Quan Z Sheng, Anne HH Ngu, and Xue Li. Things of Interest Recommendation by Leveraging Heterogeneous Relations in the Internet of Things. ACM Transactions on Internet Technology (TOIT), 16(2):9, 2016. 
18. Lina Yao, Quan Z Sheng, Anne HH Ngu, Xue Li, and Boualem Benattalah. Unveiling Correlations via Mining Human-Thing Interactions in the Web of Things. ACM Transactions on Intelligent Systems and Technology (TIST), 8(5):62, 2017.

19. Lina Yao, Quan Z. Sheng, and Schahram Dustdar. Web-based Management of the Internet of Things. IEEE Internet Computing, 19(4):60-67, 2015

20. Shuai Zhang, Lina Yao, Aixin Sun, and Yi Tay. Deep Learning based Recommender System: A Survey and New Perspectives. ACM Computing Surveys (CSUR), 52(1):5, 2019.

Lina Yao is a senior lecturer in the School of Computer Science and Engineering, UNSW Australia. She has a PhD in computer science from the University of Adelaide. Contact her at lina.yao@unsw.edu.au.

Xianzhi Wang is a lecturer in School of Software, University of Technology Sydney. He has a PhD in computer science from Harbin Institute of Technology. Contact him at xianzhi.wang@uts.edu.au.

Quan Z. Sheng is a full professor and Head of Department of Computing at Macquarie University. He has a $\mathrm{PhD}$ in computer science from the University of New South Wales. Contact him at michael.sheng@mq.edu.au.

Schahram Dustdar is a full professor of computer science and head of the Distributed Systems Group, Institute of Information Systems, at the Vienna University of Technology (TU Wien). Contact him at dustdar@dsg.tuwien.ac.at.

Shuai Zhang is a PhD student in the School of Computer Science and Engineering, UNSW Australia. Contact him at shuai.zhang@student.unsw.edu.au. 